\title{
How many beds? Helping consultants to estimate their requirements
}

\author{
David St George
}

\begin{abstract}
Two studies were carried out in different districts with clinicians who were concerned about the proposed numbers of beds to be provided based on regional targets and assessments. It was found that summary statistics can obscure wide variations over time in the demand for services. Providing beds according to average demand can therefore create a shortage of beds for a large part of the year.

It is argued that the norms and targets set by regions should be seen as a starting point for negotiations rather than as the definitive answer for setting the levels of services provided. Because of their particular circumstances certain districts may need to provide more than the levels set by the region, and clinicians and community physicians can work together to provide evidence for this.
\end{abstract}

\section{Introduction}

Assessing bed requirements for different specialties is a key issue in hospital planning. Techniques have been developed for this over the years. In the NHS the regional health authorities carry out detailed calculations of the bed requirements of each district as part of the regional strategic planning.

The regional health authority generally sets "regional targets" for specialties, either as bed norms - the number of beds per 1000 population-or as activity targets such as throughput-the number of admissions per bed per year. The targets are based on the performance of districts within the region and nationally, but the levels chosen are usually well above the average for the region. In one region, for example, the target throughput is the 75th percentile of the distribution of district throughputs. The performance achieved by the "better" districts is thus used as a yardstick and the regional health authority imposes constraints on resources for the "poorer" performers to bring them into line.

Inefficient use of available resources, however, is only one reason for an apparently "poor" performance in a district. Others include a different age structure in the catchment population (for example, a large elderly population, with a higher admission rate and lower throughput); a different mix of cases; insufficient provision of community services (including those provided by local authority social services departments), which prevents early discharge of patients; or a more deprived catchment population, with higher admission rates and longer lengths of stay in hospital. If any of these reasons underlie a district's apparently poor performance then reducing its resources to force it into line with regional targets is tantamount to blaming the district for conditions over which it may have no influence. If the district is unable to improve its performance the obvious effect of a reduction in resources is to increase the size of waiting lists.

Districts must therefore take regional targets as a starting point for negotiations about achievable performance and resource requirements, rather than accept them without question. Studies are needed to determine whether departures from regional targets and resource estimates are justified in the light of a particular district's circumstances.

This paper reports two studies in different districts

that were carried out with clinicians who were not happy with estimates of bed requirements in their specialties based on the calculations of the regional health authority. In the first study the number of cots required in a special care baby unit was assessed, and in the second study the number of beds for trauma patients required in a district general hospital to compensate for the closure of a specialist accident hospital in a neighbouring district was estimated.

\section{Study A}

The district general hospital of district A had a special care baby unit with 18 cots. The unit was in a ward several floors above the maternity unit, and the region provided the capital to relocate the unit in a purpose built unit integrated with the labour ward.

The existing unit appeared to have a low occupancy ( $71 \%$ in the year of the study), and with 2900 births a year in the hospital there was apparently a high level of provision $(6 \cdot 2$ cots per 1000 births against the regional norm of 4 per 1000). The birth rate in the hospital's catchment area was predicted to go up to 3200 by 1991 , so it was decided to provide only the number of cots required in 1991 as determined by the regional norm (13 cots).

The consultant paediatricians in the district, however, were concerned that on occasions more than the 18 cots were needed, and during these times some babies had had to be admitted to other hospitals. They suggested that the admission rate to the unit was higher than average because the hospital served a relatively high risk population (the most deprived areas of two neighbouring districts). They therefore thought that a provision above the regional norm was justified.

A study of daily occupancy of the unit during a 12 month period was carried out for the paediatricians to assess the variation in the number of beds occupied and to estimate the number of cots required to cope with existing demand. During the 12 months there was a total of 4654 occupied cot-days, which gives an average of 13 occupied cots a day, or $71 \%$ occupancy of the 18 cots. Figure 1 shows the daily occupancy and the mean of 13 beds occupied. Occupancy dropped as low as five beds and rose above the official 18 cots.

Table I gives an aggregation of the year's occupancy, showing the number of days and the number of cots occupied on those days. During a large part of the year bed occupancy levels were above the average of 13 . Figure 2 shows this as a cumulative percentage, where 13 cots would be adequate for $60 \%$ of the year but not for $40 \%$ of the year ( 145 days). Figure 2 also shows that the 18 cots were above $100 \%$ occupancy for $9 \%$ of the year (33 days).

This analysis shows that a unit that had only 13 cots would not meet current demands. Indeed, if only 13 cots were provided occupancy would have exceeded $160 \%$ at times. There were 2910 births at the hospital during the year, and the regional norm of four cots per 1000 births implies that 12 cots would be able to cope with the demand placed on the unit. More cots are clearly required, and the question is how many? In specialties where services must be provided in response to variable and uncontrollable demand there is a planning convention that services must be able to cover $95 \%$ of a given period, either by providing sufficient resources all of the time or by having the capacity to expand service provision during peak periods.
NW3 2PF

David St George, MB, honorary

lecturer in community medicine 


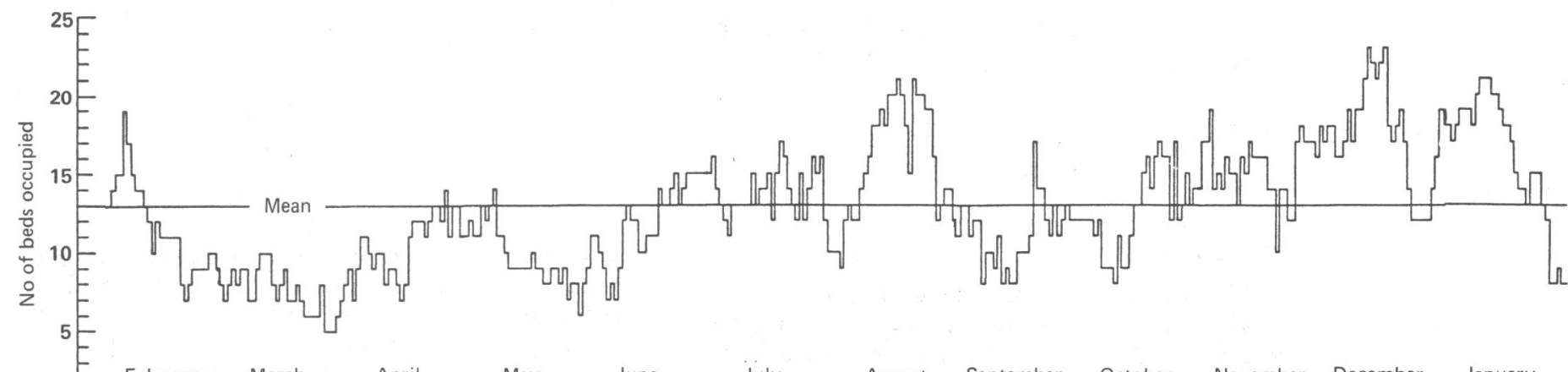

F February March April May June July August September October November December January Day intervals Day intervals

FIG 1-Daily occupancy of cots in the special care baby unit over 12 months. The horizontal line shows the mean of 13 beds occupied

Figure 2 shows that 19 cots were required to cover $95 \%$ of the study period, which is one more than the existing number of cots in the unit. Given that the birth rate is predicted to go up by about 300 by 1991, the recommendation arising from this analysis was that the level of provision in the new special care baby unit should be no less than that in the existing unit.

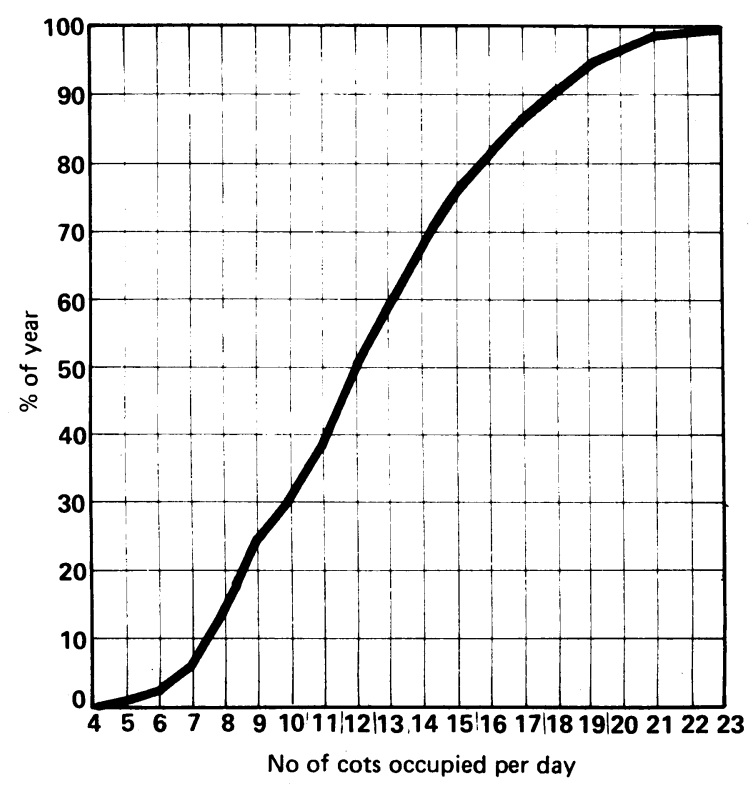

FIG 2-Cumulative occupancy of cots in the special care baby unit

The clinicians obviously accepted this analysis and its recommendations. The managers, however, were not so quick to endorse the results, primarily because of the district's severe financial crisis and the need to impose large reductions in expenditure wherever possible. Nevertheless, after lengthy negotiations the managers agreed that a reduction should not be made in the level of service provision in the new unit.

\section{Study B}

In a different region the decision was taken to close a specialist accident hospital and to reallocate its services to district general hospitals in the surrounding health districts. The regional health authority carried out a survey to determine how the workload of the accident hospital should be redistributed to the neighbouring health districts. This survey was based on the number of patients attending during one week at the specialist hospital's accident and emergency department, a tenth of whom were admitted.

The recommendations from the survey included allocating only one or two additional beds for trauma patients to district $B$ (located to the north east of the district in which the accident hospital was located), but the consultant orthopaedic surgeons in district B were not satisfied with these recommendations. The specialist accident hospital was used as an overflow for their district when there were no trauma beds available to them for admitting patients. They thought that the health authority's survey must have underestimated the use made of the accident hospital by district $B$.

A study was carried out for the orthopaedic surgeons of all admissions to the accident hospital from the resident population of district $\mathbf{B}$ during one calendar year. The district's trauma catchment population is larger than its resident population, but it was easier to identify hospital admissions by district of residence than by catchment area in the region's information system. Using data on the resident population also meant that the study would provide a conservative estimate of the use made of the accident hospital by the catchment population.

Over the year there were 177 admissions to the accident hospital from district $B$, giving an average weekly rate of 3.4 admissions. The total number of inpatient days was 2137, which gives a mean of 6 occupied beds per day. Figure 3 shows the daily occupancy and the mean. Table II gives an aggregation of bed occupancy for the year, and fig 4 shows this as a cumulative percentage. Fig 4 shows that the average of

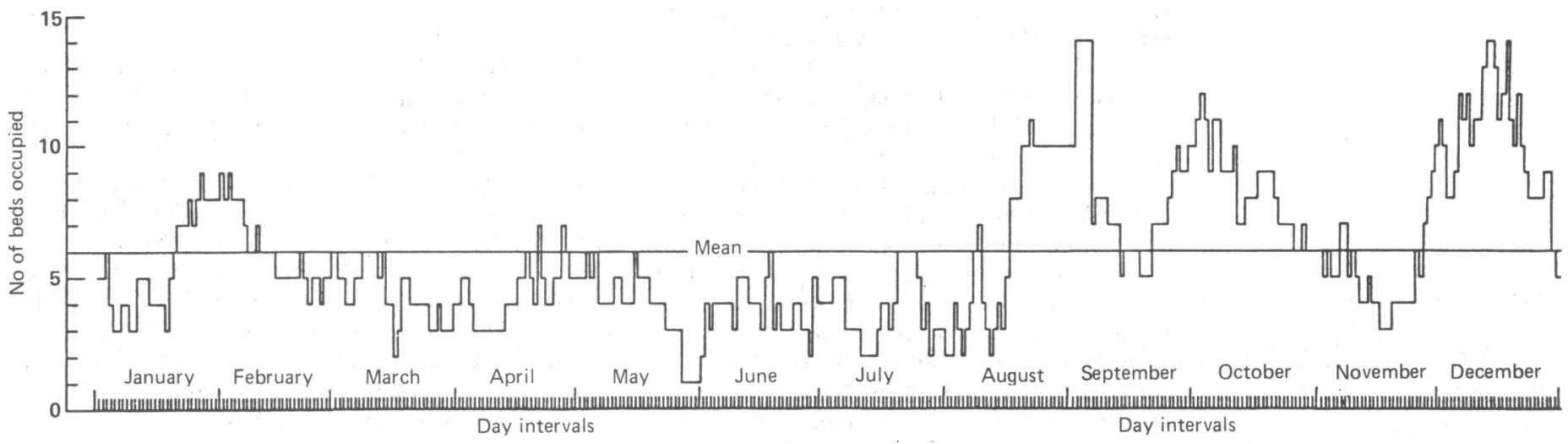

FIG 3-Daily occupancy of beds in accident hospital by resident population of district $B$. The horizontal line shows the mean of six beds occupied 


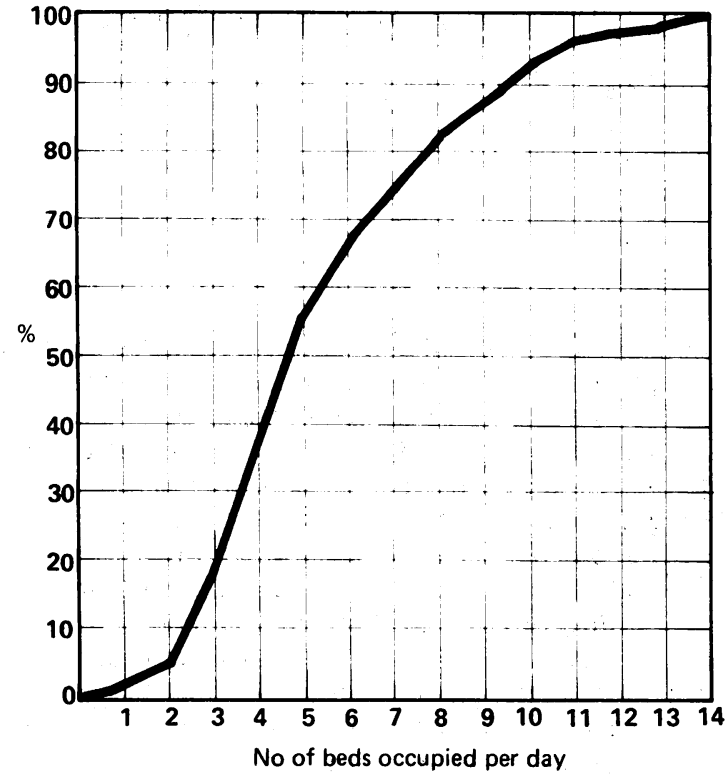

FIG 4-Cumulative occupancy of beds in accident hospital

six beds covers only $67 \%$ of the year and that to cover $95 \%$ of the year $10-12$ beds would be required.

It was recommended to the orthopaedic surgeons that district B needed 10-12 additional trauma beds to compensate for the closure of the accident hospital. The timing of this recommendation coincided with a review of acute services by the region. Using bed norms and planning populations the region had estimated that district B was roughly 24 beds short for trauma and orthopaedic patients. The study therefore helped strengthen the case for additional beds for trauma and orthopaedics, and an extra ward is now planned for the specialty.

\section{Discussion}

Most estimates of bed use are based on summary statistics only, and these can obscure considerable variation in demand. The method of analysis for the studies was chosen because it builds up a picture of bed use from day to day occupancy and shows the degree of variation in occupancy. This approach is particularly important for specialties which provide special facilities (in these cases, traction beds, neonatal cots, and highly trained staff) in response to uncontrollable demand. Ordinary beds (and staff) cannot be borrowed from other wards during peaks of demand, which means that the service must have adequate resources to cope with the busiest periods.

The findings from the studies show that basing recommendations for the provision of beds on statistical averages obscures the fact that a substantial proportion of observations can be above the mean. Choosing the average as the norm for provision therefore effectively condemns a service to a shortage of beds for part of the year. A level of bed provision must be accepted that can cope with demand throughout the year. This may result in low occupancy when the demand is low, but the number of staff could be reduced at these times.

The studies were carried out because clinicians were concerned that the regional health authority had underestimated the number of beds required, and in both cases the clinicians were justified in rejecting the estimates. Managers often say that clinicians automatically demand more beds whether or not they need them, but these findings suggest that clinicians may be right in some of their demands, and of course managers may be biased in the opposite direction. The first study shows how a regional bed norm was used in district $A$ to justify a reduction in service, but bed numbers which were below regional targets did not attract similar attention from management. For example, district $A$ had a shortage of beds for geriatric patients, and plans to increase the number would still leave the district at least 70 beds below the regional target in 1991 .

In neither study, however, was the clinical practice of the admitting clinicians questioned. In any service some admissions may be inappropriate or lengths of stay may be longer than necessary (particularly if the service has a surplus of beds). Changes in practice might therefore reduce the requirements for beds, but these studies were seen as a necessary first step in estimating bed numbers on the basis of current clinical practice. They provide a baseline for other investigations, such as clinical audit, peer review, or clinical epidemiological studies, which would show where improvements might be made.

Finally, during the present funding crisis in the NHS financial considerations increasingly dominate decisions made by health authorities about what levels of services should be provided. Planning services on need now seems like a utopian dream, and decisions are made more often in terms of what can be afforded on inadequate budgets. Health authorities should nevertheless make decisions with the full knowledge of the service levels required to meet the needs of their communities. The growing shortfall in meeting these needs should be monitored, documented, and the results published to clarify the depth of the crisis and to provide evidence for a more realistic level of funding.

(Accepted 11 fuly 1988)

\section{ANY QUESTIONS}

A woman of 40 has had depression now largely controlled by amitriptyline but she does suffer from episodes of anxiety and depression that seem to be related to menstruation. Is this a recognised pattern and what treatment is advised?

The nature of premenstrual or paramenstrual symptoms remains uncertain. A clear relation has been established between psychiatric morbidity and premenstrual complaint. ${ }^{1}$ The reasons for this association are obscure but it may be that psychiatric ill health primes or sensitises a woman to premenstrual fluctuations that might otherwise have been tolerated or ignored. It is also well recognised that patients with affective disorders may experience paramenstrual exacerbation of their symptoms.

The first question must be, is the depressive disorder being effectively treated and should the dosage of antidepressant be increased or supplemented with lithium carbonate? If the distress is so severe as to justify direct treatment of the premenstrual disturbance dydrogesterone $10-20 \mathrm{mg}$ during the premenstrual phase can be tried or the patient referred to a gynaecologist or endocrinologist.-SYDNEY BRANDON, professor of psychiatry, Leicester

1 Clare AW. Premenstrual syndrome. In: Russell GFM, Hersov LA, eds Handbook of psychiatry. Vol 4. The neuroses and personality disorders. Cambridge: Cambridge University Press, 1983:306-10.

\section{Correction}

Infection control revisited: dilemma facing today's bronchoscopists

We apologise for the editorial error that resulted in the publication of an incorrect correction (6 August, p 411) to the article by $\mathrm{Dr}$ $P$ J V Hanson and others (16 July, p 185). The authors' correction should have read, "In the $B M \mathcal{F}$ of the 16 July we stated that the prevalence of HIV is doubling over 10 months. We should have stated that the incidence of cases of AIDS is doubling every 10 months, and we apologise for this error, which occurred during manuscript revision." 